\title{
Reviewers 2018
}
K. Abbot-Smith
R. Abu-Zhaya
F. Adani
S. Adlof
J. Aguado-Orea
N. Akhtar
K. Alcock
S. Allen
C. Altman
S. Armon-Lotem
R. Bakeman
L. Barak
J. Barlow

A. Bean

H. Behrens

E. Benassi

C. Bergmann

K. Bergmann

R. Berman

T. Biberauer

A. Biemiller

T. Boerma

H. Bortfeld

C. Bowyer-Crane

S. Brandt

M. Brysbaert

K. Byers-Heinlein

N. Cabrera

O. Capirci

D. Casenhiser

M. Casillas

A. Castilla-Earls

J. Chen

S. Chiat

J. Choe

S. Choi

S. Christie

E. Clark

C. Clopper

H. Colston

S. Crain
S. Creel

S. Curtin

P. Dale

E. de Bree

J. de Jesus

J. de Jong

A. de Marchena

K. Demuth

J. Dijkstra

M. Doherty

W. Dressler

S. Durrant

M. Erbuagh

L. Fabiano-Smith

L. Farran

A. Fecica

H. Ferguson

S. Fichman

C. Fiestas

P. Fikkert

P. Fletcher

C. Floccia

D. Freudenthal

S. Fricke

R. Furman

I. Gabbatore

J. Ganger

M. Garraffa

A. Gavarro

F. Genesee

J. Gerard

M. Giezen

J. Gilkerson

T. Göksun

S. Gonzalez-Fuente

N. Gonzalez-Gomez

D. Gooch

A. Goodwin

T. Goro

J. Goslin

K. Graf-Estes
S. Graham

J. Grinstead

M. Groen

P. Hadley

E. Haman

J. K. Hamlin

C. Hamman

P. Hansen

N. Havron

K. Hawthorne

C. Hendricks

M. Hickmann

E. Hoff

G. Hollich

R. Holt

C. Hudson Kam

H. Hwang

Hyun-Joo Song

J. Iverson

D. Jackson

E. Johnson

G. Jones

H. Joseph

M. Kalashnikova

P. F. Kan

L. Kapa

M. Kehoe

B. Kelly

S. Kennison

T. Keren-Portnoy

E. Kidd

M. Kirjavainen-Morgan

M. Kline

E. Ko

A. A. Koc

J.-R. Kuipers

A. Küntay

J. Kuppersmith

F. Lacerda

C. Laing

S. A. Lee

(c) Cambridge University Press 2018 

C. Letts
C. O’Toole
D. Simard
C. Lew-Williams
J. Olson
N. Simms
F. $\mathrm{Li}$
L. Onnis
B. Skarabela
A. Lieberman
M. Ota
D. Lillo-Martin
O. Oudgenoeg-Paz
T. Sorensen Duncan
L. Lima
A. Owen van Horne
F. Southwood
C. Lleó
S. Özcalişkan
L. Sparaci
M. Lobo
R. Padilla-Reyes
G. Stam
L. Lustigman
A. Papafragou
S. Steele
A. MacLeod
J. Patterson
J. Stewart
M. MacRoy-Higgins
P. Paul
S. Stoll
A. McCabe
R. Paul
M. McGillion
A. Perez-Leroux
R. Mar
P. Perniss
C. Marshall
L. Pierce
E. Masur
J. Pine
A. Matsuo
G. Pinto
R. Mayberry
C. Potter
R. Mazuka
B. Prathanee
S. Stolt
K. Meints
R. Prevas
N. Meir
P. Prevost
H. Meister
C. Quam
J. Menjivar
L. Richland
K. Syrett
T. Tatsumi
A.-M. Tessier
A. Theakston
E. Thomas
P. Thompson
M. Thothathiri
V. Tompkins
S. Tsuji
K. Twomey
Y. Uchikoshi Tonkovich
R. Merkley
S. Ritchie
K. Messenger
K. Roskos
D. Miller
M. Rowe
S. Unsworth
V. Valian
L. Vanormelingen
S. Vasishth
S. Rvachew
J. Verhagen
G. Mirabella
L. Samuelson
M. Vihman
J. Morford
T. Sano
G. Morgan
A. Sansavini
P. Vogt
A. L. Santos
M. Vulchanova
A. Morgenstern
L. Sayahi
P. Muentener
R. Schmale
F. Warneken
I. Mundy
L. Naigles
P. Schneider
A. Weisleder
R. Weist
J. Whalen
K. Nakamura
S. Schroeder
K. White
C. Narayan
F. Schwarz
Xi Chen
E. Nilsen
A. Seidl
M. Nippold
M. Sénéchal
Xiangjun Deng
R. Nordlinger
Y. Shirai
C. Yoshinaga-Itano
Yuanyuan Wang 